\title{
When a Stroke is not Just a Stroke: Escherichia coli Meningitis with Ventriculitis and Vasculitis: A Case Report
}

\author{
Barbara Ribeiro ${ }^{1}$, Peter Bishop ${ }^{1}$, Sima Jalili2 \\ 1 Intensive Care Unit, Colchester General Hospital, East Suffolk and North Essex NHS Foundation Trust, United \\ Kingdom \\ ${ }^{2}$ Microbiology Department, Colchester General Hospital, East Suffolk and North Essex NHS Foundation Trust, United \\ Kingdom
}

\begin{abstract}
Introduction: Community-acquired Escherichia coli ventriculitis is considered a rare condition. Central nervous system (CNS) infection due to gram-negative bacilli is usually associated with previous neurosurgical interventions. The recent publication of cases of Escherichia coli meningitis and ventriculitis suggests its prevalence may be underestimated by the literature. Case presentation: A case of community-acquired Escherichia coli CNS infection on a 58 year old patient presenting with altered consciousness but without neck stiffness, nor significant past medical history is reported. Imaging and lumbar puncture findings suggested a complex case of meningitis with associated ventriculitis and vasculitis. Escherichia coli was later identified in cultures. Subsequent multi-organ support in Intensive Care was required. The patient was treated with a prolonged course of intravenous antimicrobials guided by microbiology, resulting in some neurological recovery. The main challenges encountered in the management of the patient were the lack of clear recommendations on the duration of treatment and the potential development of multi-resistant organisms. Conclusion: Bacterial central nervous system infections can have an atypical presentation, and an increasing number of cases of community-acquired ventriculitis have been reported. Early consideration should be given to use magnetic resonance imaging to help guide treatment. A long course of antibiotics is often required for these patients; however, the optimal duration for antimicrobial treatment is not well defined.
\end{abstract}

Keywords: meningitis, ventriculitis, stroke, Escherichia coli, urinary tract infection, cerebral vasculitis, ceftriaxone

Received: 20 February 2019 / Accepted: 17 January 2020

\section{INTRODUCTION}

Pyogenic ventriculitis is defined as the presence of suppurative fluid in the cerebral ventricular system [1,2]. It is usually a complication of neurosurgical procedures, ruptured brain abscesses, or head injury [1-3]. Cases of community-acquired ventriculitis are rare, with only a few cases due to Escherichia coli (E. coli) reported in the literature[2,3]. Bacteria can cause an acute septic process in the brain and the surrounding meninges, leading to cerebral vasculitis [4]. Mortality associated with $E$. coli central nervous system (CNS) infections is high (47\%), and it can be greater than $85 \%$ when associated with specific risk factors [5]. The emergence of extended-spectrum beta-lactamase (ESBL) producing Enterobacteriaceae also poses a significant challenge to the management of these cases [6,7].

\section{CASE PRESENTATION}

A 58-year old Caucasian British female presented to the emergency department (ED), Colchester General Hospital, Colchester, UK in October 2018, after she was found unresponsive by relatives. She had complained of sudden onset headache the night before admission and had attended the same ED days previously with symptoms suggesting a urinary tract infection (UTI), for which she was given unspecified oral treatment and discharged. A mid-stream urine sample obtained at that time was reported later as positive for E. coli, as were samples from previous years.

Initial findings included a Glasgow Coma Score (GCS) of 6/15 (scores of 2, 1 and 3 for eye, verbal and motor responses, respectively), right-sided weakness and facial droop. She was afebrile, without neck stiff- 
ness but myoclonic jerks in both arms were noted. Past medical and social history included anxiety, treated with sertraline. She smoked approximately thirty cigarettes per day and alcohol intake of seven units per day. There was no recent history of travel, trauma or surgery.

She was intubated in the ED and transferred to the Intensive Care Unit (ICU). The initial working diagnosis was of stroke, however considering a possible CNS infection, ceftriaxone 2 grams 12-hourly (Wockhardt UK Ltd, Wrexham, UK) and aciclovir 640 miligrams 12-hourly (Hospira UK Limited, Hurley, UK) were promptly started. Results of blood tests on admission are summarised in Table 1.

At the time of admission, a computerised tomography (CT) of the brain showed signs of a left-sided middle cerebral artery (MCA) infarct, but no hydrocephalus. On the same day, a magnetic resonance imaging (MRI) of the brain showed a right internal capsule acute infarct and debris in the ventricles suggestive of ventriculitis (Figure 1).

Two sets of blood cultures taken on the day of admission to hospital were later reported as having grown E. coli, with similar sensitivities to isolates from other fluid samples.

A lumbar puncture (LP) was performed, and cerebrospinal fluid (CSF) results are shown in Table 2.

CSF gram-stain analysis revealed that gram-negative organisms and culture results were later reported as Escherichia coli (Figures 2 and 3), susceptible to ceftriaxone. An unenhanced CT brain seven days after admission confirmed a recent right internal capsule infarct (Figure 4).

A repeat LP, on day 11, post-admission, showed marked improvement (Table 3), with no further bacterial growth.

The patient had a prolonged stay in ICU, requiring mechanical ventilation for twenty-seven days. Therefore a reliable neurological examination was only possible after sedation hold. Marked left-sided hemi-

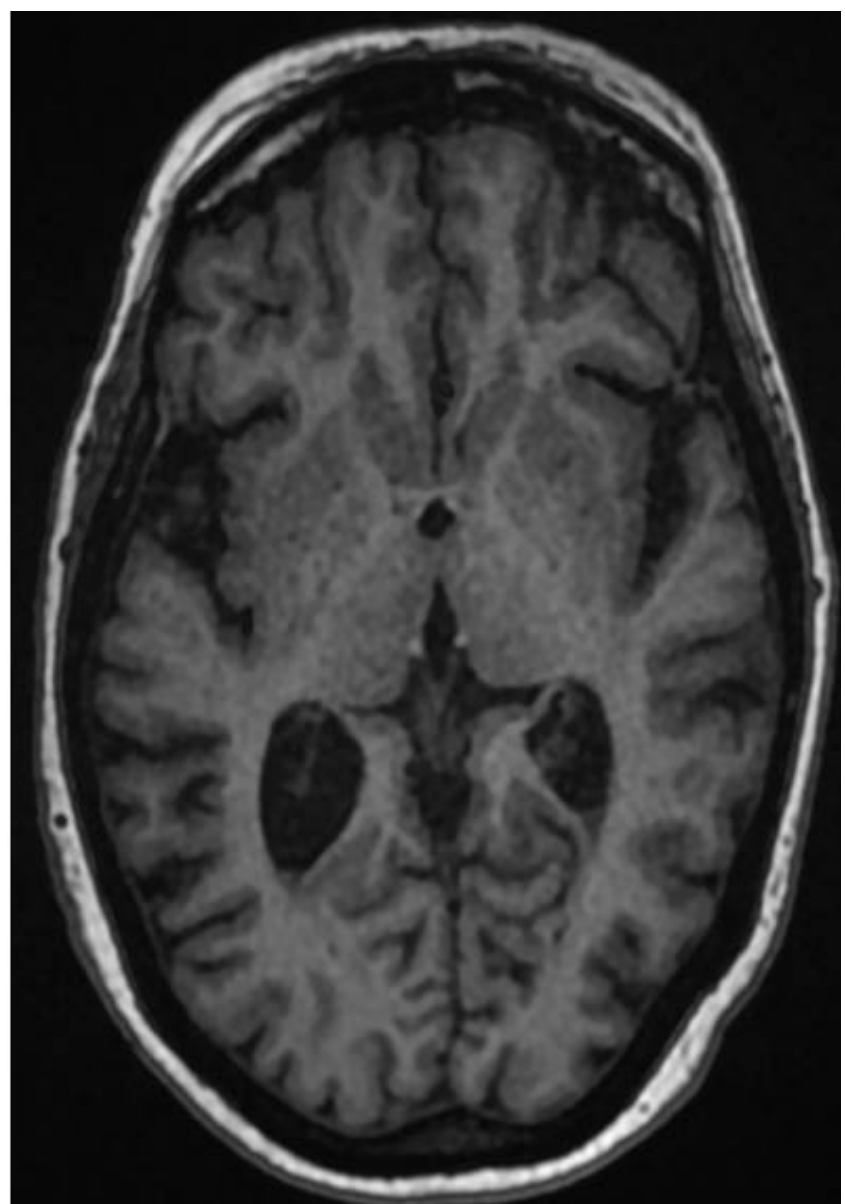

Fig. 1. Non contrast MRI brain on admission (5/10/2018) showing debris in the posterior horns of both the ventricles.

paresis was evident. Ceftriaxone was continued at the same dose and frequency until day 25 of admission, when dose was reduced to 2 grams daily. Aciclovir was stopped following the CSF microscopy report.

Strongyloides, human immunodeficiency virus (HIV), Hepatitis B and C serologies were reported negative. Renal tract ultrasound showed no evidence of hydronephrosis or collection. A transthoracic echocardiogram was reported as normal.

On day 27 of hospital admission, an MRI brain with contrast, showed persistent features of ventricu-

Table 1. Results of relevant blood tests (on admission day)

\begin{tabular}{lcc} 
Test & Result & Normal range \\
White cell count & $9.810^{9} / \mathrm{L}$ & $4-1110^{9} / \mathrm{L}$ \\
Neutrophils & $8.810^{9} / \mathrm{L}$ & $2-7.510^{9} / \mathrm{L}$ \\
Lymphocytes & $0.210^{9} / \mathrm{L}$ & $1-410^{9} / \mathrm{L}$ \\
Platelets & $6210^{9} / \mathrm{L}$ & $135-45010^{9} / \mathrm{L}$ \\
C-reactive protein & $643 \mathrm{mg} / \mathrm{L}$ & $0-5 \mathrm{mg} / \mathrm{L}$ \\
Creatinine & $232 \mu \mathrm{mol} / \mathrm{L}$ & $45-84 \mu \mathrm{mol} / \mathrm{L}$ \\
Urea & $19.7 \mathrm{mmol} / \mathrm{L}$ & $2.5-7.8 \mathrm{mmol} / \mathrm{L}$ \\
\hline
\end{tabular}




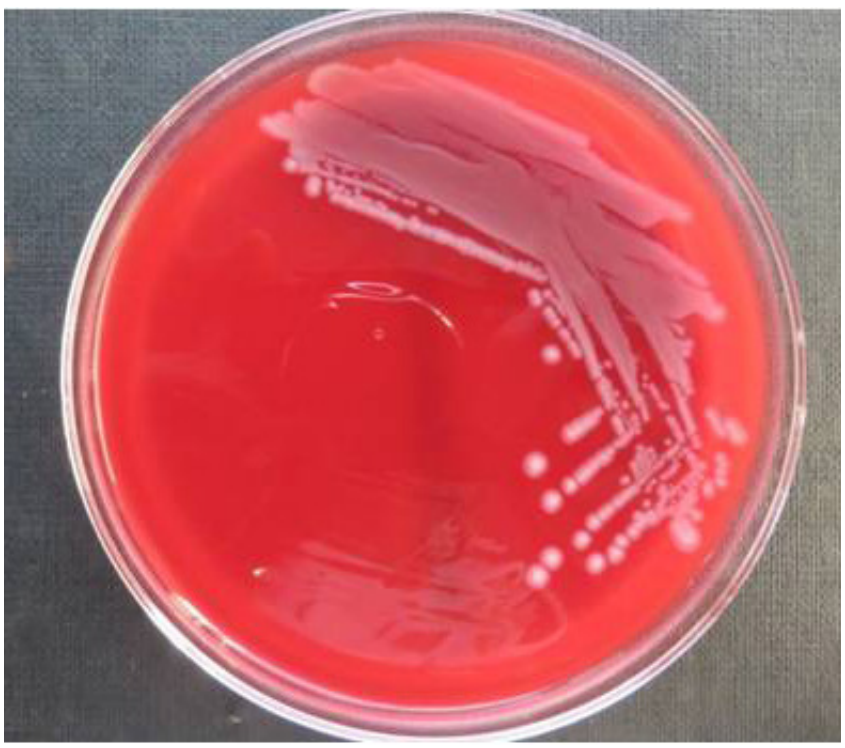

Fig. 2. E. coli colonies in blood agar.

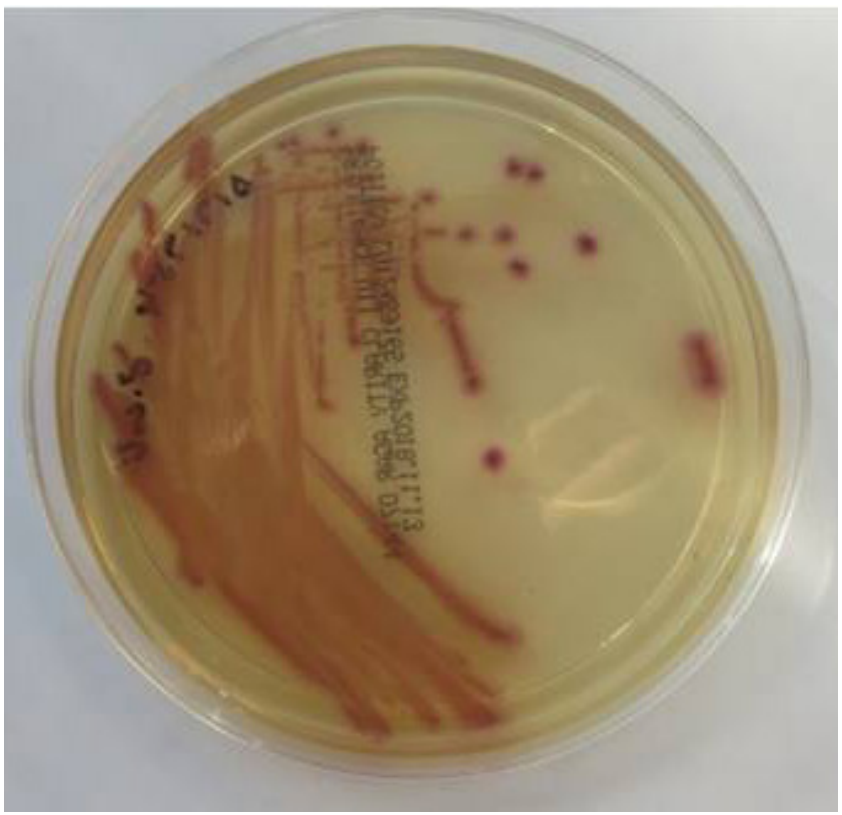

Fig. 3. E. coli colonies in chromogenic agar.

litis, a subacute infarct in the right internal capsule and trapped occipital horn of the right lateral ventricle (Figure 5). A tracheostomy was performed due to

Table 2. LP results (admission day)

\begin{tabular}{lcc} 
CSF appearance & \multicolumn{2}{c}{ CSF results } \\
& White cell count & $1568010^{6} / \mathrm{L}$ \\
& Polymorphs & $95 \%$ \\
Lymphocytes & $5 \%$ \\
Turbid & Red blood cells & $220106 / \mathrm{L}$ \\
& Protein & $4.26 \mathrm{~g} / \mathrm{L}$ \\
Glucose & $0.4 \mathrm{mmol} / \mathrm{L}$ \\
& Serum glucose & $7.0 \mathrm{mmol} / \mathrm{L}$ \\
\hline
\end{tabular}

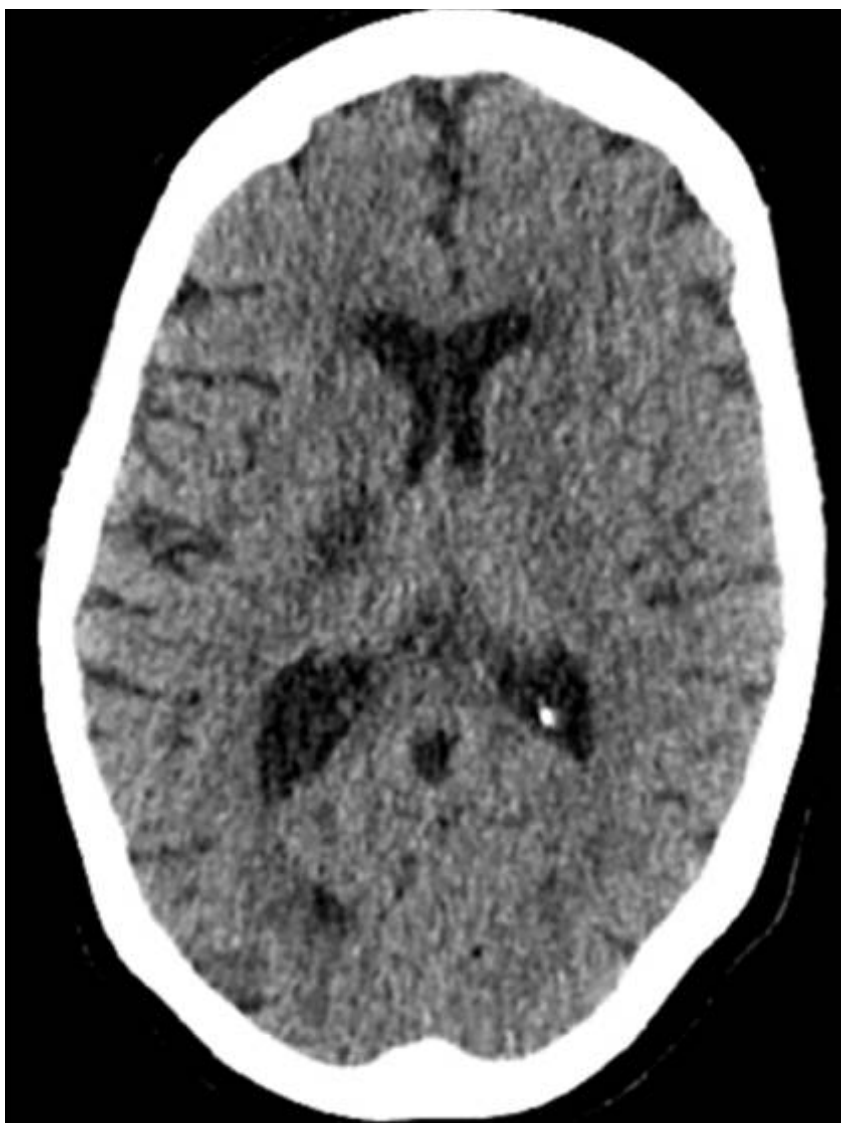

Fig. 4. Non contrast CT brain (day 7 post admission, $12 / 10 / 2018$ ) showing a low attenuation area in the posterior limb of the right internal capsule, in keeping with a recent infarct.

marked global weakness. The patient was successfully decannulated in ICU and stepped-down to the Stroke ward 29 days after hospital admission, requiring intensive physiotherapy and neuro-rehabilitation. On day forty-one post-admission, an MRI brain showed a mild reduction of a ring-enhancing lesion in the right lateral ventricle, but persistent ventricular debris (Figure 6).

The patient was discharged to a rehabilitation facility after fifty-eight days in the hospital and eight weeks of intravenous antibiotics. Follow-up imaging at four

Table 3. LP results (day 11)

\begin{tabular}{lcc} 
CSF appearance & \multicolumn{2}{c}{ CSF results } \\
& White cell count & $1410^{6} / \mathrm{L}$ \\
Polymorphs & $30 \%$ \\
Clear and & Lymphocytes & $70 \%$ \\
colourless & Red blood cells & $110^{6} / \mathrm{L}$ \\
& Protein & $0.55 \mathrm{~g} / \mathrm{L}$ \\
& Glucose & $4.1 \mathrm{mmol} / \mathrm{L}$ \\
& Serum glucose & $6.8 \mathrm{mmol} / \mathrm{L}$
\end{tabular}


months showed significant regression of the findings previously noted.

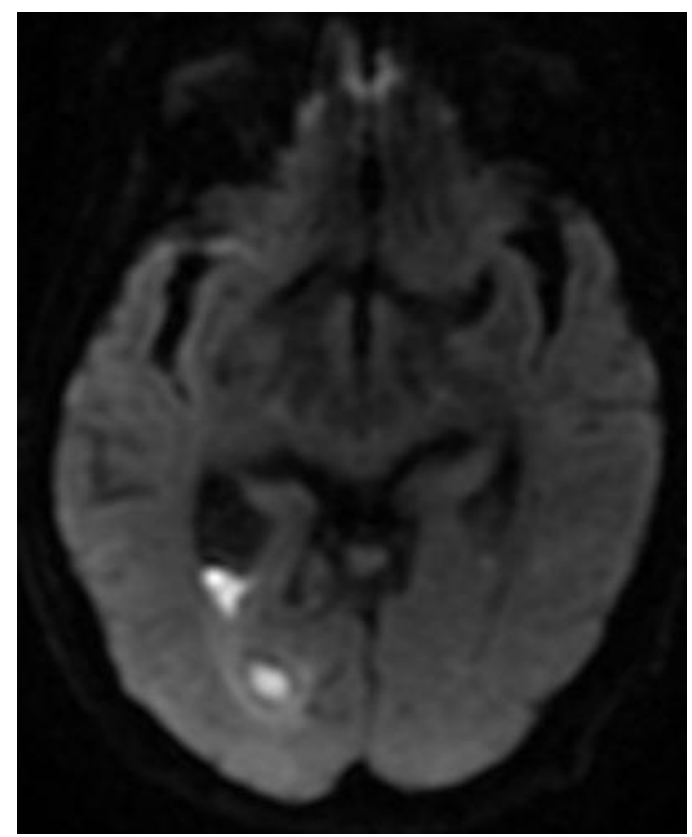

Fig. 5. MRI brain with contrast (Day 27, 2/11/2018) showing diffusion restriction in the posterior horn of the right lateral ventricle with trapped occipital horn.

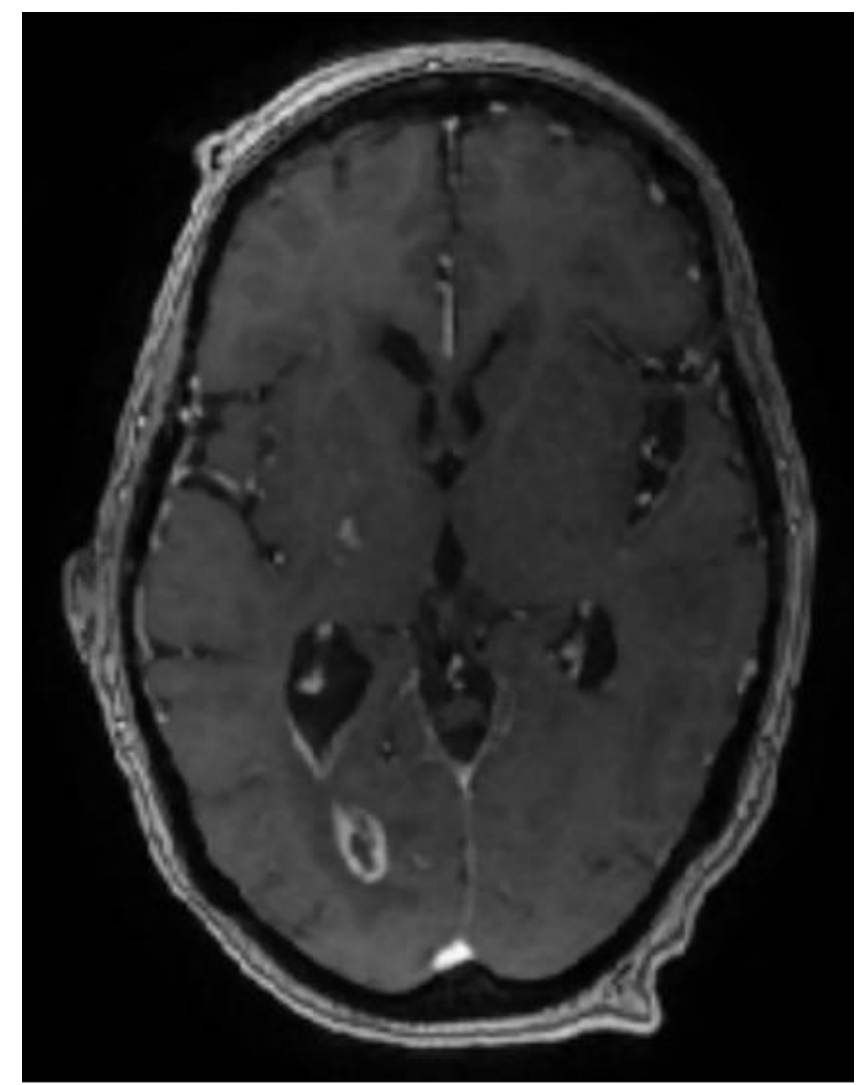

Fig. 6. MRI brain with contrast (Day 41, 16/11/2018) showing mild reduction in the size of the ring-enhancing lesion in the occipital horn of the right lateral ventricle.

\section{DISCUSSION}

Pyogenic ventriculitis is a known complication of meningitis and especially following neurosurgical interventions (e.g. shunts), trauma or CSF leak $[3,5]$. Spontaneous, community-acquired gram-negative bacilli CNS infections are reported as rare, representing $8.7 \%$ of all cases of meningitis. E. coli was reported in nearly $42 \%$ of cases [5]. A recent literature review suggested the main risk factors for community-acquired E. coli meningitis are chronic alcoholism and cirrhosis, diabetes mellitus, disseminated strongyloidiasis, HIV and chronic obstructive pulmonary disease. The same review suggested bacteraemia is the most common route of dissemination, followed by UTI and pneumonia.

E. coli is a well-known commensal in the human gastrointestinal (GI) tract $[8,9]$. These strains rarely cause disease, unless the host is immunocompromised or there is GI barrier incompetence due to peritonitis.

Three main categories of $E$. coli pathotypes have been described, causing enteric/diarrhoeal disease, urinary tract infections and sepsis/meningitis [8,9]. UTIs are the commonest extra-intestinal infections. Uropathogenic E. coli (UPEC) causes approximately $80 \%$ of UTIs, but it can also cause septicaemia [8-10]. Cases of meningitis and sepsis-associated E.coli (MNEC) have become increasingly common [8-10]. E. coli pathotypes share common mechanisms, including effective colonisation of the mucosa, evasion of the host's immune system, multiplication and host damage. They can also acquire genetic elements that ensure their adaptation to new niches $[9,10]$. UPEC migrates from the GI tract and enters the genitourinary tract. Adhesion to the uroepithelium is facilitated by fimbrial adhesion $\mathrm{H}$ (fimH), and interaction with integrins allows internalisation of bacteria that can then enter the bladder and ascend to the kidneys. Toxins, such as haemolysin, can damage the renal epithelium, and once the glomeruli and tubules are affected, bacteria can enter the circulation [8-10]. In order to cause meningitis, bacteria must cross the blood-brain barrier and proteins such as the outer membrane protein $\mathrm{A}(\mathrm{OmpA})$ and $\mathrm{K} 1$ capsule play a role in causing damage to the brain tissue $[8,9,11]$. The formation of a 'biofilm' contributes to immune evasion and act as a reservoir for recurrent infections $[8,10]$, whereas genomic plasticity contributes to the development of antibiotic resistance [11].

Recent case reports and reviews on primary ventriculitis question the true prevalence of the condition 
$[2,6]$. As clinical presentation can be non-specific, the diagnosis relies essentially on neuroimaging. The current patient did not present with neck stiffness, which is consistent with findings reported by Gronthoud et al. (2017)[3] and Kasimahanti et al. (2018)[6].

A high level of suspicion is needed, and an MRI of the brain is the most sensitive tool for early diagnosis [2]. The presence of irregular debris on MRI is specific for pus, according to Fukui et al. (2001) [12]. Recently, Vale et al. (2019) [13] published a case of communityacquired E.coli meningitis in a patient with spondylodiscitis and discoid lupus erythematosus. The current case differs from that one in so far as the current patient did not have any significant co-morbidities. As CSF, blood and urine cultures grew the same microorganism, they lead us to hypothesise that a UTI was the likely source of $E$. coli infection, with later systemic dissemination.

Cerebral vasculitis has been reported as a complication of both pneumococcal meningitis and meningococcal ventriculitis [2], supporting the current findings. Meningeal inflammation can cause infiltration of inflammatory cells within the vessel walls, leading to vessel damage [4]. This report appears to be the first documented case of $E$. coli meningitis/ventriculitis with associated vasculitis.

The different vascular territories affected, left MCA on initial imaging, right internal capsule on subsequent scans, possibly reflected the evolution of the inflammatory response and CNS infection.

There are no specific recommendations for the management of patients with community-acquired ventriculitis [2,5]. An early neurosurgical opinion should be sought; however, the optimal duration for antimicrobial treatment is not well defined [2,3]. Given the potential high mortality and morbidity, longer duration of antibiotics should be considered (6-12 weeks) [2, 5].

Decision-making, during the management of the current patient, was multidisciplinary, and the teams involved included neurosurgery, critical care, microbiology and stroke. Persistent evidence of intra-ventricular debris on imaging was the primary concern and rationale for a long course of antibiotics.

\section{CONCLUSION}

This case report illustrates the unique features of a case of community-acquired E. coli meningitis with ventriculitis and cerebral vasculitis, in a patient with no sig- nificant co-morbidities. UTIs are recognised risk factor, and the belief was that this was the initial condition leading to disseminated E. coli disease. Obtaining early samples for culture, especially CSF, is vital to the identification of the causative agent and guide treatment. Prompt treatment is required, given the severity of the disease. A multidisciplinary approach is essential to the management of such complex cases.

Universal agreement and recommendation regarding the duration of antibiotic treatment in such cases is required.

\section{ACKNOWLEDGEMENT}

Case report and supportive imaging published with written consent of the patient.

Bacterial culture images kindly provided by the Microbiology Department, Colchester Hospital.

Neuroimaging published with agreement from the Radiology Department,Colchester Hospital.

\section{DONFLICTS OF INTEREST}

None to declare.

\section{DEFERENCES}

1. Akhaddar A: Pyogenic Ventriculitis. In Akhaddar, A:Atlas of Infections in Neurosurgery and Spinal Surgery.Cham: Springer AG. 2017, pp.105-110

2. Lesourd A, Magne N, Soares A et al. Primary bacterial ventriculitis in adults, an emergent diagnosis challenge: report of a meningococcal case and review of the literature. BMC Infect Dis. 2018;18:226.

3. Gronthoud F, Hassan I, Newton P. Primary pyogenic ventriculitis caused by Neisseria meningitidis: case report and review of the literature. JMM Case Rep. 2017;4(1)

4. Jacobi C, Wildemann B, Wengenroth M.: Cerebral Vasculitis. In Hähnel, S: Inflammatory Diseases of the Brain.Leipzig: Springer. 2009,pp.19-38

5. Bichon A, Aubry C, Dubourg G et al.Escherichia coli spontaneous community-acquired meningitis in adults: A case report and literature review. Int J Infect Dis. 2018;67:70-74

6. Kasimahanti R, Kandraju Satish S, Anand M. Communityacquired Escherichia coli meningitis with ventriculitis in an adult- A rare case report. Journal of Intensive Care.2018;6:63.

7. Zeiler FA, Silvaggio J. ESBL Escherichia coli ventriculitis after aneurysm clipping: A rare and difficult therapeutic challenge. Case Rep Neurol Med. 2015; 694807

8. Kaper JB, Nataro JP, Mobley HLT.Pathogenic Escherichia coli. 
70 - The Journal of Critical Care Medicine 2020;6(1)

Nature Reviews Microbiology. 2004;2:123-140

9. Croxen MA, Finlay BB. Molecular mechanisms of Escherichia coli pathogenicity.Nature Reviews Microbiology. 2010;8:26-38

10. Kudinha T: The pathogenesis of Escherichia coli urinary tract infection. In Samidou A: Escherichia coli - recent advances on physiology, pathogenesis and biotechnological applications. IntechOpen. 2017; pp45-69

11. Kim KS. Escherichia coli translocation at the blood-brain barrier.
Available online at: www.jccm.ro Infection and immunity. 2001;69(9):5217-5222.

12. Fukui MB, Williams RL, Mudigonda S. CT and MR imaging features of pyogenic ventriculitis. AJNR Am J Neuroradiol. 2001;22(8)1510-6139.

13. Vale F, Junior VLP, Casella MI, Pocas J. Community-acquired Escherichia coli meningitis and spondylodiscitis in an adult patient with discoid lupus erythematosus. IDCases. 2019;17:e00573 